\title{
BIPOLAR OUTFLOW AND TURBULENCE IN MOLECULAR CLOUDS DUE TO PROTOSTELLAR ALFVEN WAVES
}

\author{
V. Jatenco-Pereira and R. Opher \\ Instituto Astronōmico e Geofísico da \\ Universidade de São Paulo \\ C.P. 9638, 01065 São Paulo, SP \\ Brazil
}

ABSTRACT. There is strong evidence for magnetic fields in molecular clouds to be oriented parallel to the direction of bipolar outflows. We have evidence for Alfvén waves in molecular clouds from the facts that: (i) the velocity width of CO lines, $\Delta \mathrm{V}$, is approximately twice the gravitational energy, and (ii) polarization maps indicate well-defined magnetic fields. These facts indicate that $\Delta \mathrm{V}$ is wave-like and not eddy-like. We study the Alfvén wave protostellar model of JatencoPereira and Opher (1989) to explain the observed bipolar outflows and turbulence in molecular clouds. We assume that the Alfven waves are primarily dissipated by non-linear damping and examine the physical parameters necessary to produce the observed massive low-velocity bipolar outflows ( $u_{\infty} \sim 10-50 \mathrm{~km} \mathrm{~s}^{-1}$ and $\dot{\mathrm{M}} \sim 10^{-3} \mathrm{M}_{\odot} \mathrm{yr}^{-1}$ ) and turbulence in molecular clouds.

\section{INTRODUCTION}

The outflows from young stellar objects are highly energetic with kinetic energies of up to $10^{47} \mathrm{erg}$ and exhibit a wide variety of observable phenomena. Some level of collimation usually exists for the outflows, the most notable examples being massive flows of cold molecular gas (e.g. Sne11, Loren and Plambeck 1980). In general, these molecular outflows have a mass loss rate $\dot{\mathrm{M}} \sim 10^{-3}-10^{-4} \mathrm{M}_{\odot} \mathrm{yr}^{-1}$, and terminal velocity $u_{\infty} \sim 10-50 \mathrm{~km} \mathrm{~s}^{-1}$, lower than the escape velocity ( $v_{e o}$ ). Highly collimated optical jets have also been observed in $\mathrm{H}_{\alpha}$ images (e.g. Mundt and Fried 1983). A disklike structure perpendicular to the directions of the outflows has been found in redor of the central objects (e.g. Kaifu, et. a1. 1984).

\section{THE MODEL}

We study the Alfvén wave protostellar model of Jatenco-Pereira and Opher (1989), to explain the observed bipolar outflows and turbulence in molecular clouds. The magnetic structure studied was similar to the 
coronal hole geometry of the sun of an initial nonradial diverging magnetic field of area $A(r)=A\left(r_{0}\right)\left(r / r_{0}\right)$, where $A(r)$ is the cross-sectional area of the geometry at a radial distance $r, S>2$, and $r_{\max }$ for the nonradial divergence being determined by $\left(A(r) / r^{2}\right)_{\max } /\left(A\left(r_{0}\right) / r_{0}^{2}\right)=10$. For long periods $\left(10^{6} \mathrm{~s}>\mathrm{P}>2 \times 10^{4} \mathrm{~s}\right)$ collision absorption is small, and we assume that the dominant mechanism for the absorption of the Alfven waves is nonlinear damping, with the damping rate (e.g. Lagage and Cesarsky, 1983) being given by: $\Gamma_{N L} \propto \omega\left(V_{S} / V_{A}\right)\left(\rho<\delta v^{2}>/\left(B^{2} / 8 \pi\right)\right)$ and the damping length $\mathrm{L}_{\mathrm{NL}}=\mathrm{V}_{\mathrm{A}} / \Gamma_{\mathrm{NL}}$, where $\mathrm{V}_{\mathrm{S}}$ is the sound velocity, $\mathrm{V}_{\mathrm{A}}$ is the Alfven velocity, $\rho\left\langle\delta v^{2}\right\rangle$ is the energy density of the Alfven waves and $\omega$ is a characteristic Alfvén frequency.

\section{RESULTS}

In Figure 1, we show the preliminary results of this study.

Using the initial conditions of Jatenco-Pereira and Opher (1989), with a central object of $M=1 M_{\odot}$, radius $r_{0}=10^{13} \mathrm{~cm}$, surface mass density $\rho_{0}=0.257 \times 10^{-7} \mathrm{~g} \mathrm{~cm}^{-3}$, a jet temperature $\mathrm{T}=100 \mathrm{~K}$, surface magnetic field $B \sim 40 G$, surface flux of Alfvén waves $\Phi \sim 10^{9}$ ergs $\mathrm{cm}^{-2}$ $s^{-1}$, and varying the opening angle by varying, $s$, and the initial damping length, $\mathrm{L}_{\mathrm{NLO}}$, we obtain:

a) mass loss rate $\dot{\mathrm{M}} \sim 10^{-3}-10^{-4} \mathrm{M}_{\odot} \mathrm{yr}^{-1}$ and $u_{\infty} \sim 10-50 \mathrm{~km} \mathrm{~s}^{-1}$ lower than the escape velocity $\left(v_{e o}\right)$, as observed; and

b) a non-zero Alfven waves flux, far from the star ( $\left.r \sim 300 r_{0}\right)$, that could explain the turbulence in molecular clouds (Fig. 1).

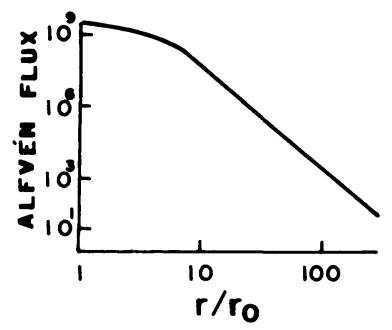

Fig. 1. Alfvēn wave flux versus $\left(r / r_{0}\right)$.

Acknowledgements

The authors would like to thank the brazilian agency CNPq for partial support.

\section{REFERENCES}

Jatenco-Pereira, V., Opher, R. (1989) Mon. Not. R. astr. Soc. 236, 1. Kaifu, S.S., Hasegawa, T., Morimoto, M., Inatami, J. et al. (1 $\overline{984})$ Astron. and Astrophys. 134, 7 .

Lagage, P.0., Cesarsky, C.J. (1983) Astron. and Astrophys. 125, 249. Mundt, R., Fried, J.W. (1983) Astrophys. J. 275, L83.

Sne11, R.L., Loren, R.B. and Plambeck, R.L. (1980) Astrophys. J. 239, L17. 